\title{
A CHARACTERIZATION OF COMPACT CONNECTED PLANAR LATTICES
}

\author{
Charles E. Clark and Carl Eberhart
}

In this paper it is proved that every topological lattice on the two-cell is topologically isomorphic (iseomorphic) to a sublattice of the product lattice $I \times I$. An explicit description of the compact connected sublattices of $I \times I$ containing $(0,0)$ and $(1,1)$ is given. These results, together with a theorem of A. D. Wallace, yield a characterization of all compact connected lattices in the plane: each is iseomorphic to a sublattice of $I \times I$.

A topological lattice is a partially ordered space $X$ with the property that every pair of elements $a, b$ of $X$ has a least upper bound, $a \vee b$, and a greatest lower bound, $a \wedge b$, so that the operations $\vee$ and $\wedge$ are continuous. A simple example of a topological lattice is the unit interval $I$ with the usual ordering. The partial order on the $n$-cell $I^{n}$ given by $\left(x_{i}\right) \leqq\left(y_{i}\right)$ if and only if $x_{i} \leqq y_{i}$ for $i=1, \cdots, n$ is a lattice ordering, in fact, it is the lattice ordering obtained by regarding $I^{n}$ as a product lattice. L. W. Anderson and A. D. Wallace have found conditions under which a lattice ordering on the $n$-cell is the product order. One can also consider the following problem: determine all lattice orderings of the $n$-cell. It is well known that the usual order is the only lattice order on the interval. In this paper the problem is considered for the two-cell. It is shown that every topological lattice on the two-cell is iseomorphic to a sublattice of the product lattice $I \times I$. This result together with a theorem of A. D. Wallace is used to prove that every compact connected lattice in the plane is iseomorphic to a sublattice of $I \times I$. Finally, an explicit description of the compact connected sublattices of $I \times I$ containing $(0,0)$ and $(1,1)$ is given.

1. Lattice orderings of the two-cell. Let $L$ be a topological lattice whose underlying space is homeomorphic to a two-cell. Since $L$ is compact, $L$ has a unique minimum element 0 and a unique maximum element 1 . It is known [1] that 0 and 1 lie on the boundary of $L$ and that the boundary arcs $T$ and $E$ determined by 0 and 1 are maximal chains in $L$ and that $T$ and $E$ generate $L$ in the sense that $L=T \vee E=T \wedge E$. In this section we prove that $L$ is iseomorphic to a sublattice of $I \times I$. The proof requires several lemmas.

LEMmA 1. Let $p, q \in L . \quad$ If $(p \wedge T) \cap T=(q \wedge T) \cap T$, then either 
$p \wedge T \subset q \wedge T$ or $q \wedge T \subset p \wedge T$.

Proof. We first assume that $p, q \in E$ and that $p \leqq q$. If $p=0$, then $p \in q \wedge T$. Suppose $p>0$ and that $p \notin q \wedge T$. It is well known that $p \vee T$ and $q \wedge T$ are arcs from $p$ to 1 and from $q$ to 0 respectively. Since $L$ is a 2-cell, it must follow that $(p \vee T) \cap(q \wedge T) \neq \square$. Let $z \in(p \vee T) \cap(q \wedge T)$ and let

$$
x=\sup \{(q \wedge T) \cap T\}=\sup \{(p \wedge T) \cap T\} .
$$

Then $z=p \vee t$ for some $t \in T$. If $t \leqq x$, then by the definition of $x$, we would have $p \vee t=p$. Hence $t>x$. But now the inequality $t \leqq z \leqq q$ implies that $q \wedge t=t \in q \wedge T$ which contradicts the choice of $x$.

Now let $p$ and $q$ be arbitrary elements of $L$ and choose $e, f \in E$ such that $p \in e \wedge T$ and $q \in f \wedge T$. This is possible since $E \wedge T=L$. If either of $p$ or $q$ is an element of $T$, then the lemma is trivial. For suppose $p \in T$. Then

$$
p \wedge T=(p \wedge T) \cap T=(q \wedge T) \cap T \subset q \wedge T .
$$

We may now assume that $p, q \notin T$. We contend that $(e \wedge T) \cap T=$ $(p \wedge T) \cap T=(f \wedge T) \cap T=(q \wedge T) \cap T . \quad$ To establish the first equality, let $t \in(e \wedge T) \cap T$. Then since $e \wedge T$ is a chain and $p, t \in e \wedge T$, either $p \leqq t$ or $t \leqq p$. Suppose $p \leqq t$. Then for some $t_{1} \in T, p=e \wedge t_{1}=\left(e \wedge t_{1}\right) \wedge t=(e \wedge t) \wedge t_{1}=t \wedge t_{1} \in T$, which is a contradiction. Therefore $t \leqq p$ and $t \in(p \wedge T) \cap T$. Now suppose $t \in(p \wedge T) \cap T$. Then $t \leqq p \leqq e$ implies that $t \in(e \wedge T) \cap T$. This proves the first equality; the last equality is proved similarly. From the first part of the proof, we conclude that either $e \wedge T \subset f \wedge T$ or $f \wedge T \subset e \wedge T$. Suppose $f \wedge T \subset e \wedge T$. Then $p \wedge T$ and $q \wedge T$ are subchains of $e \wedge T$, so either $p \wedge T \subset q \wedge T$ or $q \wedge T \subset p \wedge T$.

For $x \in T$, we define $C_{x} \subset E$ by $C_{x}=\{h \in E \mid x=\sup \{(h \wedge T) \cap T\}$.

Lemma 2. The set $C_{x}$ is closed for all $x \in T$.

Proof. We consider the nontrivial case where $C_{x} \neq \square$. From the continuity of $\wedge$ it follows that the set $\{h \in E \mid x \in h \wedge T\}$ is closed. Let $e^{\prime}=\inf \{h \in E \mid x \in h \wedge T\}$; then $x \in e^{\prime} \wedge T$ and $e^{\prime} \leqq e$ for all $e \in C_{x}$. If $t \in\left(e^{\prime} \wedge T\right) \cap T$ and $t>x$, then for $e \in C_{x}$, we would have $t \leqq e^{\prime} \leqq e$ and hence $t \in(e \wedge T) \cap T$ contradicting the fact that $e \in C_{x}$. Hence $x=\sup \left\{\left(e^{\prime} \wedge T\right) \cap T\right\}$ and $e^{\prime} \in C_{x}$.

Let $h_{n} \in C_{x}, n=1,2, \cdots$, and let $h_{n} \rightarrow h$. Then $e^{\prime} \leqq h_{n}$ for each $n$ and by Lemma 1, we have that $e^{\prime} \wedge T \subset h_{n} \wedge T$ for all values of $n$ and therefore $e^{\prime} \wedge T \subset h \wedge T$. Let $x^{\prime}=\sup \{(h \wedge T) \cap T\}$. Then 
$x^{\prime} \geqq x$ since $x \in(h \wedge T) \cap T$. We have that $e^{\prime}, x^{\prime} \in h \wedge T$ and so one of the inequalities $x^{\prime} \leqq e^{\prime}, e^{\prime} \leqq x^{\prime}$ must hold. If $x^{\prime} \leqq e^{\prime}$, then $x^{\prime} \in(e \wedge T) \cap T$ which implies that $x^{\prime} \leqq x$ and hence $x^{\prime}=x$ and $h \in C_{x}$. If $\mathrm{e}^{\prime} \leqq x^{\prime}$, let $e^{\prime}=h \wedge t$ for $t \in T$. Then

$$
e^{\prime}=e^{\prime} \wedge x^{\prime}=(h \wedge t) \wedge x^{\prime}=\left(h \wedge x^{\prime}\right) \wedge t=x^{\prime} \wedge t \in T .
$$

This involves a contradiction unless $e^{\prime}=0$. However, if $e^{\prime}=0$, then $x=0$ and $h_{n} \wedge T=0$ for all values of $n$; hence $n \wedge T=0$ and $h \in C_{x}$. This completes the proof of the lemma.

We now define relations $\mathscr{C}$ and $\mathscr{V}^{n}$ on $T$ as follows: for $a, b \in T$,

$$
\begin{aligned}
& a \mathscr{\mathscr { C }} b \equiv a \in e \vee T \text { if and only if } b \in e \vee T \text { for all } e \in E . \\
& a \mathscr{Y} b \equiv a \in e \wedge T \text { if and only if } b \in e \wedge T \text { for all } e \in E .
\end{aligned}
$$

Lemma 3. The relations $\mathscr{\mathscr { C }}$ and $\mathscr{Y}$ are closed congruences on $T$.

Proof. It is easy to see that $\mathscr{H}$ and $\mathscr{Y}^{-}$are congruences on $T$. We will show that the relation $\mathscr{V}$ is closed. A dual argument will show that $\mathscr{C}$ is closed.

Let $a_{n} \rightarrow a, b_{n} \rightarrow b$ with $a_{n}, b_{n} \in T$ and $a_{n} \mathscr{y} b_{n}$ for each $n$. Assume that $a \leqq b$. If $h \in e \wedge T$ for $e \in E$, it follows trivially that $a \in e \wedge T$. Suppose $a \in e \wedge T$ for $e \in E$. Let $x=\sup \{(e \wedge T) \cap T\}$; then $a \leqq x$. If $a<x$, then for $n$ sufficiently large, $a_{n}<x$ and hence $a_{n} \in e \wedge T$. Since $a_{n} \mathscr{Y} b_{n}$ we must have $b_{n} \in e \wedge T$ for $n$ sufficiently large and therefore $b \in e \wedge T$. This gives $a \mathscr{y} b$.

We now assume that $a=x$ and let $f=\sup C_{x}$. This sup exists since $C_{x}$ is closed by Lemma 2. If $f=1$, then $a=b=1$. Suppose $f<1$ and let $f_{m} \rightarrow f$ where $f_{m} \in E, f_{m}>f$ for $m=1,2, \cdots$. Let $y_{m}=\sup \left\{\left(f_{m} \wedge T\right) \cap T\right\}$. Then since $f_{m} \notin C_{x}, y_{m}>a$. Thus for fixed $m$, there exists a positive integer $N_{m}$ such that if $n \geqq N_{m}$, then $a_{n}<y_{m}$, or $a_{n} \in f_{m} \wedge T$. Therefore $b_{n} \in f_{m} \wedge T$ for $n \leqq N_{m}$. We conclude that $b \in f_{m} \wedge T$ for each positive integer $m$ and hence $b \in f \wedge T$. But $a=\sup \{(f \wedge T) \cap T\}$ and hence $b \leqq a$. Therefore $a=b$.

Lemma 4. Let $e \in E$ and let $x=\sup \{(e \wedge T) \cap T\}, e^{\prime}=\sup C_{x \prime}$ $x^{\prime}=\inf V_{x}$ where $V_{x}$ denotes the congruence class modulo of which contains $x$. Then $\left\{z \mid x^{\prime} \leqq z \leqq e^{\prime}\right\} \subset e^{\prime} \wedge T$.

Proof. If $z \in T$, then $z \leqq x \leqq e^{\prime}$ implies $z=e^{\prime} \wedge z \in e^{\prime} \wedge T$. Suppose $z \notin T$ and let $f \in E$ such that $z \in f \wedge T$. If $f=0$, then $z=0 \in e^{\prime} \wedge T$. Suppose $f>0$. We have $x^{\prime} \leqq z \leqq f$ and therefore $x^{\prime} \in f \wedge T$ and since $x^{\prime} \mathscr{V}^{\prime} x, x \in f \wedge T$. If $t \in(f \wedge T) \cap T$, then $t \in(z \wedge T) \cap T$ since 
$z \wedge T \subset f \wedge T$ and $z \notin T$. From the inequality $t \leqq z \leqq e^{\prime}$ we conclude that $t \in\left(e^{\prime} \wedge T\right) \cap T$ and hence $t \leqq x$. Hence $x=\sup \{(f \wedge T) \cap T\}$ and by Lemma 1 we have $f \wedge T \subset e^{\prime} \wedge T$ and therefore $z \in e^{\prime} \wedge T$.

Lemma 5. If $e, f \in E$ and $p \in[(f \vee T) \cap(e \wedge T)] \backslash T$, then $\{p\}=$ $(f \vee T) \cap(e \wedge T)$.

Proof. Suppose $p^{\prime} \in(f \vee T) \cap(e \wedge T)$. Then either $p^{\prime} \leqq p$ or $p^{\prime} \geqq p$ and in either case it is easily seen that $p^{\prime} \notin T$ since $p \notin T$. Assume that $p^{\prime} \leqq p$ and let $x=\sup \{(e \wedge T) \cap T\}$. Then since $p, p^{\prime} \notin T, x=\sup \{(p \wedge T) \cap T\}=\sup \left\{\left(p^{\prime} \wedge T\right) \cap T\right\}$. Since $p^{\prime} \leqq p$ on $f \vee T$, we have that $p \in p^{\prime} \vee T$ so that $p=p^{\prime} \vee t$ for some $t \in T$ and since $x=\sup \left\{\left(p^{\prime} \wedge T\right) \cap T\right\}$, it follows that $t \geqq x$. But $t \leqq p \leqq e$ implies that $t \in(e \wedge T) \cap T$ and so $t \leqq x$. Hence $t=x$ and $p=$ $p^{\prime} \vee x=p^{\prime}$.

Lemma 6. Let $x \in T$ and let $x^{\prime}=\sup V x$. Then $C_{x^{\prime}} \neq \square$.

Proof. The set $\{h \in E \mid x \in h \wedge T\}$ is closed by the continuity of $\wedge$ and is nonempty since $x \in 1 \wedge T$. Let $e=\inf \{h \in E \mid x \in h \wedge T\}$. Then $x \in e \wedge T$ and since $x \mathscr{V}^{\prime} x^{\prime}$ it follows that $x^{\prime} \in e \wedge T$. Let $x^{\prime \prime}=$ $\sup \{(e \wedge T) \cap T\}$. Then $x^{\prime \prime} \leqq x^{\prime}$. Suppose $h \in E$ and $x \in h \wedge T$. Then $h \geqq e$ by the definition of $e$ and since $x^{\prime \prime} \in e \wedge T$ it follows that $x^{\prime \prime} \in h \wedge T$. On the other hand, if $x^{\prime \prime} \in h \wedge T$ for some $h \in E$, then $x \in h \wedge T$ since $x \leqq x^{\prime \prime}$. Therefore $x \mathscr{V}^{\prime} x^{\prime \prime}$ but since $x^{\prime \prime} \geqq x^{\prime}$ and $x^{\prime}=\sup \mathscr{V}^{\prime} x$, we must have $x^{\prime \prime}=x^{\prime}$. Hence $e \in C_{x^{\prime}}$.

We are now prepared to define the iseomorphism from $L$ into $I \times I$. For $p \in L$, define

and

$$
\alpha_{1}(p)=\sup \{(p \wedge T) \cap T\}
$$

$$
\alpha_{2}(p)=\inf \{(p \vee T) \cap T\} .
$$

Let $\eta_{1}, \eta_{2}$, denote the natural maps from $T$ onto $T / \mathscr{Y}=T_{1}$ and $T / \mathscr{C}=T_{2}$ respectively. Let $\phi_{1}=\eta_{1} \circ \alpha_{1}, \phi_{2}=\eta_{2} \circ \alpha_{2}$ and define

by

$$
\phi: L \rightarrow T_{1} \times T_{2}
$$

$$
\dot{\phi}=\dot{\phi}_{1} \times \dot{\phi}_{2} .
$$

THEOREM 1. If $L$ is a topological lattice which is homeomorphic to a 2-cell, then $L$ is iseomorphic to a sublattice of $I \times I$.

Proof. We will show that the map defined above is a one-to-one continuous homomorphism from $L$ into $T_{1} \times T_{2}$. The theorem then 
follows since $T_{1} \times T_{2}$ is iseomorphic to $I \times I$.

(i) The map $\phi$ is continuous. We show $\phi_{1}$ is continuous. A dual argument shows that $\phi_{2}$ is continuous.

Let $x \in T_{1}$ and let $a=\sup \eta_{1}^{-1}(x)$. Then $C_{a} \neq \square$ by Lemma 6 . Let $e=\sup C_{a}$. We claim that $\phi_{1}^{-1}[0, x]=e \wedge L$. A similar argument shows that $\phi_{1}^{-1}[x, 1]=a^{\prime} \vee L$ where $a^{\prime}=\inf \eta_{1}^{-1}(x)$. Thus the inverse under $\phi_{1}$ of a subbasic closed set is closed in $L$ and hence $\alpha_{1}$ is continuous.

Let $z \in e \wedge L$. Then $b=\sup \{(z \wedge T) \cap T\} \leqq z \leqq e$ and so $b \leqq a$. Then $\phi_{1}(z)=\eta_{1}\left(\alpha_{1}(z)\right)=\eta_{1}(b) \leqq \eta_{1}(a)=x$. Hence $z \in \dot{\phi}_{1}^{-1}[0, x]$. Now let $z \in \phi_{1}^{-1}[0, x], b=\sup \eta_{1}^{-1}\left(\phi_{1}(z)\right)$, and $f=\sup C_{b}$. Since $\phi_{1}(z) \leqq x$, then $b \leqq a$. If $z \in T$ then $z \leqq b \leqq a \leqq e$; thus $z \in e \wedge L$.

Now suppose that $z \notin T$. From the definition of $b$ we have $\eta_{1}(b)=$ $\eta_{1}\left(\alpha_{1}(z)\right)$ and hence $b \mathscr{V}^{\prime} \alpha_{1}(z)$. Therefore $\alpha_{1}(z) \leqq b$. Let $h \in E$ such that $z \in h \wedge T$. Then since $z \notin T$, it was shown in the proof of Lemma 1 that $\sup \{(z \wedge T) \cap T\}=\sup \{(h \wedge T) \cap T\}$. Therefore $\alpha_{1}(z) \in h \wedge T$ and since $b \mathscr{V} \alpha_{1}(z)$, we have $b \in(h \wedge T) \cap T$ and hence $b \in(z \wedge T) \cap T$. Then by the definition of $\alpha_{1}(z)$, we have $b \leqq \alpha_{1}(z)$. Thus $\alpha_{1}(z)=b$, and $(z \wedge T) \cap T=(f \wedge T) \cap T$. By Lemma $1, z \wedge T \subset f \wedge T$. Since $b \leqq a$, then $f \leqq e$. Hence $z \leqq f \leqq e$ implies that $z \in e \wedge L$.

(ii) $\phi$ is one-to-one. Suppose $p, p^{\prime} \in L$ such that $\phi_{i}(p)=\phi_{i}\left(p^{\prime}\right)$, $i=1,2$. We will show that $p=p^{\prime}$. We consider three cases.

Case 1. $\quad p, p^{\prime} \in L \backslash T$. Then since $\phi_{1}(p)=\eta_{1}\left(\alpha_{1}(p)\right)=\eta_{1}\left(\alpha_{1}\left(p^{\prime}\right)\right)=$ $\phi_{1}\left(p^{\prime}\right)$, we have that $\alpha_{1}(p) \mathscr{Y} \alpha_{1}\left(p^{\prime}\right)$. Choose $e, f \in E$ such that $p \in e \wedge T$ and $p^{\prime} \in f \wedge T$. Then from the proof of Lemma 1 , it follows that

$$
\sup \{(e \wedge T) \cap T\}=\sup \{(p \wedge T) \cap T\}=\alpha_{1}(p),
$$

and

$$
\sup \{(f \wedge T) \cap T\}=\sup \left\{\left(p^{\prime} \wedge T\right) \cap T\right\}=\alpha_{1}\left(p^{\prime}\right) .
$$

But since $\alpha_{1}(p) \mathscr{Y}^{\prime} \alpha_{1}\left(p^{\prime}\right)$, we must have $\alpha_{1}\left(p^{\prime}\right) \in(e \wedge T) \cap T$ and $\alpha_{1}(p) \in(f \wedge T) \cap T$. It now follows that $\alpha_{1}\left(p^{\prime}\right) \leqq \alpha_{1}(p) \leqq \alpha_{1}\left(p^{\prime}\right)$ and hence $\alpha_{1}(p)=\alpha_{1}\left(p^{\prime}\right)=\alpha_{1}(e)=\alpha_{1}(f)$. Hence by Lemma 1, either $f \wedge T \subset e \wedge T$ or $e \wedge T \subset f \wedge T$. Suppose $f \wedge T \subset e \wedge T$. Then $p, p^{\prime} \in e \wedge T$. Using a similar argument and the dual of Lemma 1 we obtain $g \in E$ such that $p, p^{\prime} \in g \vee T$. Since $p, p^{\prime} \notin T$, we conclude from Lemma 5 that $p=p^{\prime}$.

Case 2. $p, p^{\prime} \in T$. Assume $p \leqq p^{\prime}$. If $p^{\prime}=1$, then $p^{\prime} \in 1 \vee T$ and $p^{\prime} \mathscr{C} p$ implies that $p \in 1 \vee T$ and so $p=1$. Suppose $p^{\prime}<1$ and let $f=\sup \{h \in E \mid p \in h \vee T\}$. Then $f<1$. Let $f_{n} \rightarrow f$, where $f_{n} \in E$ and $f_{n}>f$ for all $n$. Then $p \notin f_{n} \vee T$ and hence $p^{\prime} \notin f_{n} \vee T$ for all $n$. Therefore if $f_{n} \vee p \in T$, then $f_{n} \vee p>p^{\prime}$, and if $f_{n} \vee p \notin T$ then 
$p=\left(f_{n} \vee p\right) \wedge p \in\left(f_{n} \vee p\right) \wedge T$ and hence $p^{\prime} \in\left(f_{n} \vee p\right) \wedge T$ since $p \mathscr{V}^{\prime} p^{\prime}$ and $f_{n} \vee p \notin T$. So $f_{n} \vee p \geqq p^{\prime}$ for all $n$. Therefore, by the continuity of $\vee, p=f \vee p \leqq p^{\prime}$. Then $p=p^{\prime}$.

Case 3. $p \notin T, p^{\prime} \in T$. Choose $e, f \in E$ such that

$$
p \in(e \wedge T) \cap(f \vee T) \text {. }
$$

Then since $p \notin T,\{p\}=(e \wedge T) \cap(f \vee T)$ by Lemma 5. Since $\phi_{1}(p)=\phi_{1}\left(p^{\prime}\right)$, we have $\sup \{(p \wedge T) \cap T\} \mathscr{Y}^{\prime} p^{\prime}$ from which follows $p^{\prime} \in p \wedge T \cap e \wedge T$. Similarly, $p^{\prime} \in f \vee T$, contradicting Lemma 5 .

(iii) $\dot{\phi}$ is a homomorphism. We will show that $\phi_{1}$ is a homomorphism with respect to $V$, Similar arguments will show that $\phi_{1}$ is a homomorphism with respect to $\wedge$ and that $\phi_{2}$ is a homomorphism with respect to $\vee$ and $\wedge$.

Let $p, p^{\prime} \in L ; x=\alpha_{1}(p)=\sup \{(p \wedge T) \cap T\}$,

$$
x^{\prime}=\alpha_{1}\left(p^{\prime}\right)=\sup \left\{\left(p^{\prime} \vee T\right) \cap T\right\},
$$

and

$$
z=\alpha_{1}\left(p \vee p^{\prime}\right)=\sup \left\{\left(\left(p \vee p^{\prime}\right) \wedge T\right) \cap T\right\} .
$$

Assume that $x \leqq x^{\prime}$. Then $x \vee x^{\prime}=x^{\prime}$ and $\eta_{1}\left(x \vee x^{\prime}\right)=\eta_{1}(x) \vee \eta_{1}\left(x^{\prime}\right)=$ $\eta_{1}\left(x^{\prime}\right)$. Then $\dot{\phi}_{1}(p) \vee \dot{\phi}_{1}\left(p^{\prime}\right)=\eta_{1}(x) \vee \eta_{1}\left(x^{\prime}\right)=\eta_{1}\left(x^{\prime}\right)$. We will show that $\phi_{1}\left(p \vee p^{\prime}\right)=\eta_{1}(z)=\eta_{1}\left(x^{\prime}\right)$, i.e., $z \mathscr{V}^{\top} x^{\prime}$.

We have that $x^{\prime} \leqq p^{\prime} \leqq p \vee p^{\prime}$, so $x^{\prime} \in\left(\left(p \vee p^{\prime}\right) \wedge T\right) \cap T$ and hence $x^{\prime} \leqq z$. If $z \in e \wedge T$ for $e \in E$, then clearly $x^{\prime} \in e \wedge T$. Now suppose $x^{\prime} \in e \wedge T, e \in E$. We consider two cases.

Case 1. $p^{\prime} \in E$. We may assume that $e=\inf \left\{h \in E \mid x^{\prime} \in h \wedge T\right\}$. If $p^{\prime} \in T$, then $p^{\prime}=x^{\prime} \in e \wedge T$. If $p^{\prime} \in T$, then choose $g \in E$ such that $p^{\prime} \in g \wedge T$. Then $x^{\prime} \leqq p^{\prime} \leqq g$ implies that $x^{\prime} \in g \wedge T$ and hence $e \leqq g$.

From Lemma $6, e=\sup C_{x^{\prime}}$. But the proof of Lemma 1 gives

$$
x^{\prime}=\sup \left\{\left(p^{\prime} \wedge T\right) \cap T\right\}=\sup \{(g \wedge T) \cap T\},
$$

and therefore $g \leqq e$. Hence $g=e$ and $p^{\prime} \leqq e$.

We will show that $p \leqq e$ also. If $p \in T$, then $p=x \leqq x^{\prime} \leqq e$. Suppose $p \in T$ and let $f=\inf \{h \in E \mid p \in h<T\}$. Then since $p \notin T$, $\sup \{(f \wedge T) \cap T\}=\sup \{(p \wedge T) \cap T\}=x \leqq x^{\prime}$ and hence $f \leqq e$. Then the inequality $p \leqq f \leqq e$ gives the desired conclusion.

We now have $p^{\prime} \leqq e, p \leqq e$; hence $p \vee p^{\prime} \leqq e$. Since $p^{\prime} \in e \wedge T$, the inequality $p^{\prime} \leqq p \vee p^{\prime} \leqq e$ and Lemma 4 gives $p \vee p^{\prime} \in e \wedge T$. Hence $z \in e \wedge T$. This concludes the proof for Case 1 .

Case 2. $p^{\prime} \in E$. If $p^{\prime} \leqq p$, then $p \vee p^{\prime}=p$ implies $x=z$. But 
then $x \leqq x^{\prime} \leqq z$ implies $x^{\prime}=z$ and so $z \in e \wedge T$.

If $p^{\prime} \notin p \wedge L$ then since

$$
x=\sup \{(p \wedge T) \cap T\} \leqq x^{\prime}=\sup \left\{\left(p^{\prime} \wedge T\right) \cap T\right\},
$$

the proof of the continuity of $\phi_{1}$ shows that $p \in p^{\prime} \wedge L$. Hence $p \vee p^{\prime}=p^{\prime}$ and again we conclude that $z=x^{\prime}$. This concludes the proof that $\phi_{1}$ is a homomorphism with respect to $\vee$, and the proof of Theorem 1.

2. Compact connected lattices in the plane. In [4] Wallace proved that a compact connected lattice $L$ which is imbeddable in the plane is a cyclic chain (in the sense of Whyburn \{5]) and that each true cyclic element is a convex sublattice and is homeomorphic to a 2-cell. Thus by Theorem 1, each true cyclic element is iseomorphic to a sublattice of $I \times I$. Let $\Delta$ denote the diagonal thread in $I \times I$. Label the true cyclic elements of $L,\left\{C_{i}\right\}_{i=1}^{\infty}$. Denote the 0 and 1 of $C_{i}$ by $a_{i}$ and $b_{i}$ respectively. Let $T$ be any maximal chain from 0 to 1 in $L$, and let $h$ be an iseomorphism from $T$ onto $\Delta$, the diagonal in $I \times I$. Then the "square" in $I \times I$ with upper right hand vertex $h\left(b_{i}\right)$ and lower left hand vertex $h\left(a_{i}\right)$ is a sublattice of $I \times I$ which is iseomorphic to $I \times I$. Hence $C_{i}$ may be imbedded in this sublattice as in Theorem 1. In this manner an iseomorphism of $L$ into $I \times I$ is determined. Thus we have proven:

THEOREM 2. Every compact connected lattice in the plane is iseomorphic to a sublattice of $I \times I$.

Finally we state an explicit description of the compact connected sublattices of $I \times I$ containing $(0,0)$ and $(1,1)$.

THEOREM 3. Let $f$ and $g$ be functions from $I$ into $I$ satisfying

(i) $f, g$ are nondecreasing, $f(0)=0, g(1)=1$,

(ii) $f(x) \leqq g(x)$ for all $x \in I$,

(iii) $f$ is continuous from the left and $g$ is continuous from the right.

Then the set $L=\{(x, y): f(x) \leqq y \leqq g(x)\}$ is a compact connected sublattice of $I \times I$ containing $(0,0)$ and $(1,1)$. Conversely, if $L$ is a compact connected sublattice of $I \times I$ containing $(0,0)$ and $(1,1)$ then there exist functions $f$ and $g$ satisfying i-iii such that

$$
L=\{(x, y): f(x) \leqq y \leqq g(x)\} .
$$

Proof. The proof is straightforward and will be omitted. The functions $f$ and $g$ alluded to in the second part are defined as follows: 


$$
\begin{array}{ll}
g(x)=\sup \{L \cap(\{x\} \times I)\} & \text { for } x \in I \\
f(x)=\inf \{L \cap(\{x\} \times I)\} & \text { for } x \in I .
\end{array}
$$

3. Comments. Edmondson has given an example of a topological lattice on a 3-cell which is nonmodular; hence this lattice is not a sublattice of $I \times I \times I[2]$. This shows that the higher dimensional analogous of Theorem 1 are false.

This the result of this paper does not hold if the term "lattice" be replaced by "semilattice" is a consequence of the results of D. R. Brown, [1], regarding semilattice structures on the two-cell.

Wallace has conjectured that every 2-dimensional compact connected lattice with no cutpoints is a two-cell. A related conjecture is that every 2-dimensional compact connected lattice can be imbedded in the plane. If this were true, the words "in the plane" in the statement of Theorem 2 could be replaced by "2-dimensional."

The authors are pleased to acknowledge their indebtedness to Professor R. J. Koch for his suggestions in the preparation of this paper.

\section{REFERENCES}

1. D. R. Brown, Topological semilattices on the two-cell Pacific J. Math. 15 (1965).

2. Don E. Edmondson, A nonmodular compact connected topological lattice, Proc. Amer. Math. Soc. 7 (1956).

3. A. D. Wallace, Factoring a lattice, Proc. Amer. Math. Soc. 9 (1958).

4. - Two theorems on topological lattices. Pacific J. Math. 7 (1957).

5. G. T. Whyburn, Analytic Topology, Coll. Pub. Amer. Math. Soc., 1942.

Received May 2, 1966. 